\title{
Perfil del cuidador del paciente con Enfermedad Renal Crónica: una revisión de la literatura
}

\author{
Elveny Laguado-Jaimes \\ Universidad Cooperativa de Colombia. Sede Bucaramanga. Colombia
}

Como citar este artículo:

Laguado-Jaimes E. Perfil del cuidador del paciente con Enfermedad Renal Crónica: una revisión de la literatura.

Enferm Nefrol. 2019 Oct-Dic;22(4):352-59

\section{Resumen}

Introducción: Los cuidadores de pacientes con enfermedad renal crónica (ERC) presentan alteraciones físicas, sociales, económicas, espirituales y emocionales, derivadas de su rol. Conocer el perfil del cuidador principal es fundamental para establecer intervenciones de apoyo y asistencia.

Objetivo: Determinar el perfil del cuidador del paciente con ERC referido en la literatura, que describa las variables sociodemográficas y el predominio de condiciones de salud relacionadas con el cuidado del familiar.

Material y Método: Se realizó una revisión narrativa de la literatura, siguiendo recomendaciones de la declaración PRISMA. Se incluyeron estudios primarios (experimentales u observacionales, cuantitativos y cualitativos), acerca de los efectos en la salud del cuidador familiar de pacientes. Se consultaron las bases de datos ProQuest, ScienceDirect, Scopus, Dialnet, Redalyc y Scielo; entre 2011 y 2018.

Resultados: Como características sociodemográficas de los cuidadores familiares, predomina el $41,66 \%$ mayor de 53 años; género femenino con 92,30\%, estado civil: casados $66,66 \%$; escolaridad primaria $26,08 \%$ y ocupación ama de casa $22,22 \%$. En referencia a los efectos en salud, los emocionales prevalecen la ansiedad y depresión, seguido de alteraciones en el sueño, que afecta la calidad de vida del cuidador.

\section{Correspondencia:}

Elveny Laguado Jaimes

E-mail: Elveny.laguado@campusucc.edu.co
Conclusión: El conocimiento del cuidador en sus variables sociodemográficas permite establecer acciones acordes a las necesidades y la intervención de efectos emocionales, mala calidad del sueño, el aislamiento social que afecta la calidad de vida del cuidador y por ende al paciente.

PALABRAS CLAVE: diálisis; hemodiálisis; calidad de vida; cuidadores; sobrecarga.

\section{Profile of caregivers of Chronic Kidney Disease patients: a review of the literature}

\section{Abstract}

Introduction: Caregivers of chronic kidney disease (CKD) patients have physical, social, economic, spiritual and emotional changes, derived from their role. Knowing the profile of the primary caregiver is essential to establish support and assistance interventions.

Objective: To determine the profile of caregivers of CKD patients referred to in the literature, which describes the sociodemographic variables and the predominance of health conditions related to family care.

Material and Method: A narrative review of the literature was carried out, following recommendations of the PRISMA statement. Primary studies (experimental or observational, quantitative and qualitative studies) about the health effects in the family caregiver of CKD patients were included. The databases ProQuest, ScienceDirect, Scopus, Dialnet, Redalyc and Scielo were consulted in the period between 2011 and 2018. 
Results: In relation to the sociodemographic characteristics of family caregivers, $41.66 \%$ are older than 53 years; $92.30 \%$ being female and $66.66 \%$ being married; with primary education in $26.08 \%$ and $22.22 \%$ of housewife occupation. In reference to the health effects, the emotional effects that prevail are anxiety and depression, followed by sleep disturbances, which affects the quality of life of the caregiver.

Conclusion: The knowledge of the caregiver's sociodemographic variables allows to establish actions according to the needs and intervention of emotional effects, poor quality of sleep, social isolation, which affects the quality of life of the caregiver, and therefore, the patient.

KEYWORDS: dialysis; hemodialysis; quality of life; caregivers; burden.

\section{Introducción}

Según datos del Fondo Colombiano de Enfermedades de alto Costo para el año 2017 en Colombia 4.124.668 pacientes tienen alguna enfermedad precursora para la enfermedad renal crónica (ERC) y de estos, 1.406.364 tienen diagnosticado algún estadio de ERC dentro de la población afiliada a las aseguradoras que reportan a este organismo $(2,85 \%$ de la población de Colombia en 2017) ${ }^{1}$.

En la insuficiencia renal, las distintas alteraciones (cambios hemodinámicos, desnutrición, anemia severa, osteodistrofias, alteraciones cardiovasculares, etc), conllevan diferentes grados de dependencia física e implican cambios en la vida familiar, tanto del paciente como del cuidador primario. Este último se ve involucrado en una serie de problemas de orden físico, psíquico, emocional, social o económico que es Ilamado carga del cuidador. Producto de las demandas del cuidado del familiar, el cuidador requiere apoyo ante la responsabilidad y tareas del cuidado, además de en las tareas domésticas, administración en el hogar y movilidad en el entorno, durante la mayor parte del día, sin retribución económica².

Con frecuencia los cuidados son proporcionados por personas que pasan desapercibidas al no pertenecer al equipo de salud. Su ayuda es insustituible y estas actividades van más allá de solo proporcionar afecto al enfermo hospitalizado. Este cuidador familiar, que está unido por parentesco, asume la responsabilidad de aten- der las necesidades de la persona hospitalizada, la cual presenta una situación de dependencia: el tener o no el conocimiento es una dificultad para el cuidado del familiar con enfermedad renal ${ }^{3}$.

El acto del cuidado implica una interacción humana de carácter interpersonal, intencional y único, que sucede en un contexto sociocultural: el cuidar de otro conlleva la intervención para la supervivencia social y física de las personas en condición de dependencia. Es un proceso intersubjetivo y simétrico, en el cual ambos, quien es cuidado y el que cuida, establecen una relación recíproca y afectiva, que parte del reconocimiento del otro como ser humano ${ }^{4}$.

Otra de las problemáticas para el cuidador, radica en que frecuentemente es ignorado por la familia y por el equipo terapéutico: predomina el conocimiento de las patologías crónicas y sus cuidados de salud, pero requieren un refuerzo educativo en el tiempo y mejorando la comunicación con el personal sanitario. El conocimiento de las características y necesidades del cuidador permite determinar las intervenciones pertinentes que apoyen al cuidador incidiendo en resultados positivos para sí mismos y para la persona cuidada, ${ }^{5,6}$.

El cuidador familiar en su labor diaria acumula diferentes cargas, éstas con el tiempo inciden en manifestaciones físicas (aumento de carga de trabajo, problemas sexuales), sociales (aislamiento, disminución de actividades sociales, cambios en la relación con el paciente), económicas (restricciones), espirituales y emocionales (como sentimientos de tristeza, agotamiento e impotencia, entre otros) $)^{6,7}$.

Para establecer las intervenciones y actividades con el fin de prevenir la aparición de las repercusiones sobre su calidad de vida, es preciso identificar las situaciones de riesgo $0^{6}$. Por tanto, involucrar a los cuidadores, identificando el perfil del cuidador principal (incluyendo sus características sociodemográficas y efectos del cuidado), es fundamental para establecer intervenciones de apoyo y asistencia para sobrellevar las acciones de cuidado de su familiar.

El presente trabajo pretende determinar el perfil del cuidador del paciente con ERC referido en la literatura, que describa las variables sociodemográficas (edad, género, nivel educativo y ocupación), y el predominio de condiciones de salud relacionadas con el cuidado del familiar. 


\section{Material y Método}

Se realizó una revisión narrativa de la literatura. Para la revisión se siguieron las recomendaciones de la declaración PRISMA. Se incluyeron estudios primarios (experimentales u observacionales, cuantitativos y cualitativos), acerca de los efectos en la salud del cuidador familiar de pacientes con ERC, con los siguientes criterios de inclusión: periodo de revisión (2011 a 2018); idioma español, inglés y portugués; que incluyeran una descripción de las variables sociodemográficas de cuidadores de pacientes ERC y los efectos en su salud derivados de este rol.

Se consultaron las bases de datos ProQuest, ScienceDirect, Scopus, Dialnet, Redalyc y Scielo. Adicionalmente se realizó una revisión en el Repositorio Institucional de la de la Universidad Autónoma de México y Perú. Se utilizaron las siguientes palabras clave:

Español: Cuidadores, Pacientes, Insuficiencia Renal Crónica, Variables sociodemográficas, sobrecarga, diálisis, hemodiálisis, trasplante renal, dependencia, calidad de vida.

- Inglés: patients, kidney, caregivers, characterization sociodemographic, Hemodialysis patients; Family caregivers; Quality of sleep; Quality of life, caregivers, burden.

- Portugués: Cuidadores, Pacientes, Insuficiência Renal Crônica, caracterização sociodemográfica, Pacientes de hemodiálise, Transplante de Rim, dependencia, Qualidade de Vida.

Se utilizaron combinaciones de términos con el operador booleano "OR" para términos dentro del mismo dominio de la pregunta PICO, y el operador "AND" para combinar términos/búsquedas de cada dominio. El proceso de localización y selección de estudios según se muestra en las Figura $\mathbf{1}$.

Para la síntesis de datos se realizó un análisis descriptivo de los artículos publicados incluidos en la búsqueda incluyendo: país de publicación, características sociodemográficas del cuidador familiar (edad, género, nivel de estudios), condiciones de salud de los cuidadores (dimensión afectada y patología).

\section{Resultados}

En la revisión de los artículos se identificaron 27 estudios, de los siguientes países: España con 26,92\%, México 19,23\%, Colombia, Turquía, Brasil y Asia 7,69\%, Chile, Cuba, Reino Unido, Estados Unidos, Arabia y Perú: 3,84\% artículos. De los anteriores, un 53,84\% fueron estudios cuantitativos, un $42,30 \%$ cualitativos y un $3,84 \%$ de tipo mixto.

El cuidador del familiar que está diagnosticado con enfermedad renal, se constituye en una persona que apoya el cuidado de forma directa y se involucra en diferentes

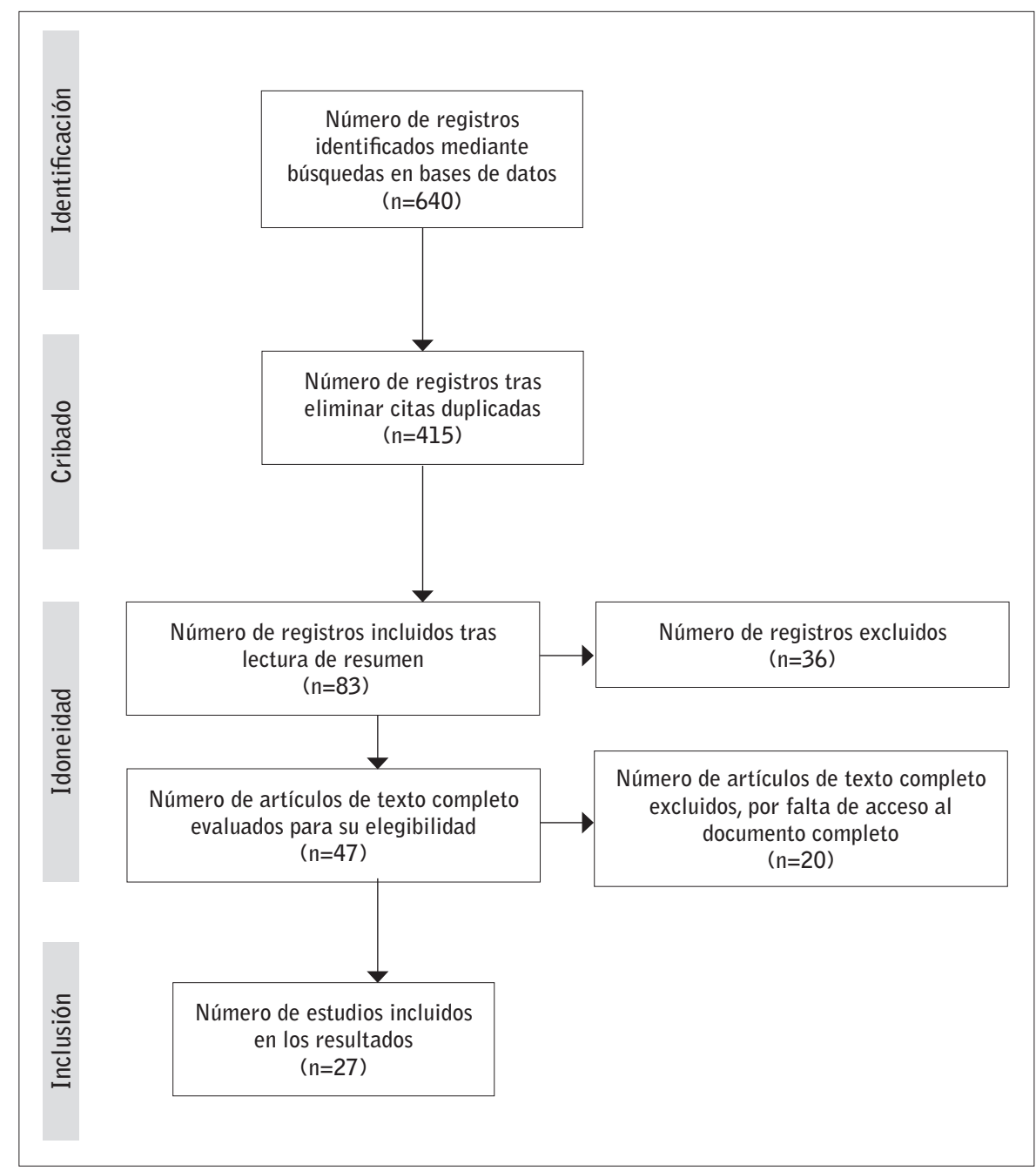

Figura 1. Flujograma de estrategia de búsqueda y proceso de selección de estudios. 
situaciones relacionadas con las respuestas positivas o del paciente; el conocimiento del cuidador que se describe a continuación parte de las variables sociodemográficas, estas pueden o no estar relacionadas con los posibles efectos en las diferentes dimensiones que afectan la salud del cuidador.

\section{Características Sociodemográficas}

En la Tabla 1 se muestra el perfil del cuidador de cada uno de los estudios incluidos, en función del estado civil mayoritario. De los estudios revisados el $92,30 \%$ muestran el predominio del género femenino para los cuidadores, frente a un $7,69 \%$ del género masculino. Respecto al estado civil, el $66,66 \%$ de los estudios refiere casados mayoritariamente. En referencia a la edad el $41,66 \%$ es mayor de 53 años, comparado con un 20,83\% menores de 40 años. Con respecto a la escolaridad el $44,44 \%$ de los estudios no incluyen esta variable, pero predomina el $22,22 \%$ para la escolaridad de primaria y secundaria. De forma similar, la variable ocupación no se recoge en el
$48,14 \%$ de los estudios revisados: entre los que describen esta variable, las amas de casa son mayoritarias en el $25,92 \%$ de los estudios revisados.

\section{Efectos en los Cuidadores}

A partir de los estudios cualitativos, surgen categorías como la carga del cuidado, disminución de la calidad de vida o el afrontamiento inefectivo ${ }^{4}$. Otras categorías encontradas fueron: sensación de impotencia sobre la salud y el futuro de los pacientes en diálisis, sobrecarga en los cuidadores, alto grado de estrés y la fatiga, que conduce al agotamiento físico, biológico y emocional; e incertidumbre ${ }^{19}$.

De los resultados obtenidos, se identifican aquellos predominantes por porcentajes, clasificados según la dimensión afectada: emocional, física, social, espiritual y laboral.

Dimensión Emocional: predomina la ansiedad en cuatro estudios $^{8,12,16,24}$ y la depresión en cinco estudios $7,8,12,16,25$. Otro estudio refiere efectos como miedo, angustia, soledad

Tabla 1. Características sociodemográficas de los cuidadores según su estado civil.

\begin{tabular}{|c|c|c|c|c|}
\hline ESTADO CIVIL MAYORITARIO & AUTOR (AÑo) & EDAD MEDIA / GENERO & Nivel de estudios & OCUPACIÓN \\
\hline Casados & $\begin{array}{l}\text { Jarrillo }(2014)^{4} \\
\text { Romero }(2014)^{6} \\
\text { Velázquez }(2014)^{8} \\
\text { Arechabala }(2011)^{7} \\
\text { Noble }(2012)^{9} \\
\text { Bayoumi }(2014)^{10} \\
\text { Eterovic }(2015)^{11} \\
\text { Ambriz }(2014)^{3} \\
\text { Gil }(2013)^{12} \\
\text { Morton }(2012)^{13} \\
\text { Contreras }(2013)^{14} \\
\text { Angulo }(2016)^{2} \\
\text { Méndez }(2012)^{15} \\
\text { Çelik }(2012)^{16} \\
\text { Williams }(2015)^{17} \\
\text { Da Silva Barreto }(2011)^{18}\end{array}$ & $\begin{array}{l}44 \text { años/Femenino: } 100 \% \\
\text { Sin dato/Femenino: } 76,9 \% \\
40 \text { a } 59 \text { años/Femenino: } 87,5 \% \\
66 \text { años/Femenino: } 75 \% \\
50-60 \text { años/Femenino: } 68,4 \% \\
\text { >40 años/Femenino: } 70 \% \\
45-64 \text { años/Femenino: } 88,1 \% \\
39 \text { años/Femenino: } 86,3 \% \\
67 \text { años/Femenino: (sin dato) } \\
61 \text { años/Femenino: (sin dato) } \\
64 \text { años/Femenino: } 64 \% \\
41,7 \text { años/Femenino: } 79,7 \% \\
40 \text { años/Femenino: } 66,1 \% \\
46,1 \text { años/Femenino: } 62 \% \\
73 \text { años/Masculino: } 100 \% \\
48 \text { años/Femenino: } 100 \%\end{array}$ & $\begin{array}{l}\text { Primaria } \\
\text { Tecnológica } \\
\text { Secundaria } \\
\text { Sin dato } \\
\text { Sin dato } \\
\text { Primaria } \\
\text { Sin dato } \\
\text { Primaria } \\
\text { Primaria } \\
\text { Sin dato } \\
\text { Sin dato } \\
\text { Primaria } \\
\text { Sin dato } \\
\text { Secundaria } \\
\text { Sin dato } \\
\text { Sin dato }\end{array}$ & $\begin{array}{l}\text { Ama de Casa } \\
\text { Ama de casa } \\
\text { Trabajador } \\
\text { Sin dato } \\
\text { Desempleado } \\
\text { Desempleado } \\
\text { Sin dato } \\
\text { Ama de casa } \\
\text { No trabajan } \\
\text { Sin dato } \\
\text { Sin dato } \\
\text { Ama de casa } \\
\text { Sin dato } \\
\text { Sin dato } \\
\text { Trabajadores } \\
\text { Desempleado }\end{array}$ \\
\hline Solteras & $\begin{array}{l}\text { Rivero-García }(2015)^{19} \\
\text { López }(2015)^{20} \\
\text { Shdaifat }(2012)^{21} \\
\text { Guerra }(2016)^{26}\end{array}$ & $\begin{array}{l}36 \text { años/Femenino: } 87 \% \\
48 \text { años/Femenino: } 87 \% \\
\text { Sin datos } \\
18-35 \text { años/Femenino: sin dato }\end{array}$ & $\begin{array}{l}\text { Sin dato } \\
\text { Bachillerato Completo } \\
\text { Secundaria } \\
\text { Escolaridad alta }\end{array}$ & $\begin{array}{l}\text { Sin dato } \\
\text { Amas de casa } \\
\text { Trabajadores activos } \\
\text { Labores de hogar }\end{array}$ \\
\hline Sin dato & $\begin{array}{l}\text { Aguilera }(2016)^{22} \\
\text { Rodríguez }(2017)^{27} \\
\text { Alnazly }(2016)^{28} \\
\text { Arechabala }(2012)^{29} \\
\text { Aguilera }(2012)^{23} \\
\text { Avsar et al }(2015)^{24} \\
\text { Carmona }(2015)^{25}\end{array}$ & $\begin{array}{l}54 \text { años/Femenino } \\
51-60 \text { años/Femenino: } 44 \% \\
<30 \text { años/Masculino: } 63 \% \\
\text { 50+/-16,1 años/Femenino: } 75 \% \\
\text { Sin datos } \\
43,1 \text { años/Femenino: } 58,8 \% \\
66,5 \text { años/Femenino: } 67 \%\end{array}$ & $\begin{array}{l}\text { Sin dato } \\
\text { Secundaria } \\
\text { Sin dato } \\
\text { Sin dato } \\
\text { Primaria } \\
\text { Secundaria } \\
\text { Sin dato }\end{array}$ & $\begin{array}{l}\text { Sin dato } \\
\text { Sin dato } \\
\text { Sin dato } \\
\text { Sin dato } \\
\text { Ama de casa } \\
\text { Sin dato } \\
\text { Sin dato }\end{array}$ \\
\hline
\end{tabular}

Sin dato: El estudio no aporta resultados. 
e irritabilidad, culpa, desesperanza y resentimiento ${ }^{8}$. El rol del cuidador se considera muy estresante, lo cual es asociado a síntomas depresivos y afectación de la salud en general ${ }^{29}$. Otro aspecto que implica esta dimensión es el afrontamiento como cuidador, que requiere autocontrol, distanciamiento, resolución de problemas y una reevaluación positiva ${ }^{28}$.

Dimensión Física: se revisa en los estudios los efectos en esta dimensión desde la valoración de sobrecarga. Un estudio refiere su población como altamente sobrecargado físicamente ${ }^{8}$; en cuatro estudios indican ausencia de sobrecarga ${ }^{2,8,11,28}$, uno sobrecarga intensa ${ }^{14}$, uno carga moderada ${ }^{25}$ y uno carga moderada a severa ${ }^{10}$. En un estudio valorando la calidad de vida su variable de función física muestra afectación ${ }^{23}$; ahora en un estudio que reporta la carga intensa según la escala de $\mathrm{Za}$ rit, agregan que esto contribuye a un deterioro físico y mental, dado que hay poco tiempo para sí mismos, el asumir múltiples roles, responsabilidades y alteraciones en su salud, como consecuencia del cuidado ${ }^{26}$.

Dimensión Social: para un estudio predominan conflictos sociales y económicos, no disponer de tiempo libre y problemas familiares ${ }^{8}$, un estudio con datos de repercusiones negativas en su vida social ${ }^{3}$, reducción de vínculos sociales, poca motivación para nuevas relaciones o mantenerlas vigentes ${ }^{26}$.

Dimensión Espiritual: los participantes del estudio tienen una baja probabilidad de afectarse en esta dimensión ${ }^{6}$; de igual forma en un estudio cualitativo los cuidadores refieren que al asumir el rol de cuidador la compensación es personal y esto lo relacionan con la espiritualidad ${ }^{20}$.

Dimensión Laboral: en el cuidador se disminuyen las oportunidades para avanzar como profesional o superarse $^{26}$; un estudio reporta pérdidas a nivel laboral ${ }^{3}$.

\section{Discusión}

Sobresale en los estudios la mujer en el cuidado. En un estudio de revisión hacen referencia desde la cultura e historia, el reconocimiento de la mujer como cuidadora a nivel de la familia ${ }^{30}$ de igual forma el predominio de las mujeres, hijas en el cuidado de los adultos mayores $^{31}$.

El nivel de escolaridad en su mayoría fue de educación primaria y secundaria; contrario al estudio de personas con ECNT: Enfermedades Crónicas no transmisibles y los cuidadores familiares, en el que predomina un nivel de conocimiento universitario ${ }^{32}$.

En la revisión se observa predominio de efectos a nivel emocional; similar al estudio de revisión en el que predominan en los estudios alteraciones como la ansiedad, temor, angustia, miedo entre otros ${ }^{35}$; los familiares con la enfermedad se perciben como una carga familiar ante las demandas emocionales que implica su cuida$\mathrm{do}^{36}$; sin embargo, en un estudio mencionan que los cuidadores altamente resilientes manifiestan una menor tendencia a síntomas depresivos y de ansiedad, constituyéndose en un factor protector ${ }^{32}$; otro estudio refiere que desde el bienestar sicológico es sentirse útili37.

En el bienestar físico, en la revisión el nivel sobrecarga reporta diferentes niveles de sobrecarga, con mayor frecuencia la ausencia, similar al reporte de percepción de no sobrecarga ${ }^{38}$; sin embargo, en un estudio muestra una percepción de sobrecarga intensa en el $71 \%$ de los cuidadores $^{33}$.

El abordaje de las personas con enfermedades que implican dependencia y su cuidador familiar, requiere el fortalecimiento de habilidades y de reconocer la relación de cuidado que impacta la salud del paciente y que se constituye en duradera y a largo plazo; involucrar las acciones de cuidado en los planes identificados para los pacientes y que fortalezcan la salud de éste binomi $0^{34}$.

En lo referente a la dimensión social, es similar a estudios en los que muestra el impacto en las relaciones sociales ${ }^{39}$, de igual manera en la revisión de estudios reconocen que la tarea del cuidador impacta su vida y se refleja específicamente en el tiempo libre, su vida laboral y profesional, salud y bienestar ${ }^{35}$; cabe resaltar que para un cuidador es importante contar con redes de apoyo familiares, amistades o recursos de la socie$\mathrm{dad}^{26}$.

Finalmente es importante resaltar que el cuidado como esencia del ser, se condiciona por normas y expectativas que hacen que su compromiso sea un deber; por lo anterior el conocimiento de las necesidades y motivaciones de los cuidadores se constituye en un referente que soporta la relevancia de involucrar a éste cuidador en el proceso de intervención de los pacientes con enfermedad renal crónica, puesto que sus funciones se relacionan con el bienestar, recuperación y estabilidad del enfermo ${ }^{40}$. 


\section{Conclusiones}

Mediante la revisión de resultados de diferentes estudios, el perfil del cuidador familiar se describe como predominio de mujeres cuidadoras, estado civil casadas, edad media de 36-59 años, un nivel de estudios bajo: primaria y ocupación: amas de casa.

En los reportes de identifica que la falta de conocimientos generales sobre la enfermedad y sus cuidados por parte del cuidador, conlleva a unas manifestaciones físicas y psicológicas que alteran la calidad de vida del cuidador y con impacto en el paciente.

Podemos llegar a determinar que la sobrecarga depende de la edad de pacientes y cuidadores, los múltiples roles que desempeña este cuidador, el bajo nivel educativo, su ocupación no remunerada y la falta de apoyo del sistema en salud, que adolece de apoyo para que esta carga en el desempeño del rol se presente.

Como efectos emocionales en los cuidadores se destacó la ansiedad, la depresión y la mala calidad del sueño; en los efectos a nivel socioeconómico están la carga ligera percibida, la carga intensa y el aislamiento social; y en los efectos físicos se encuentra con mayor prevalencia el bienestar físico o de salud alterado, fatiga y agotamiento y cambios en el apetito.

Recibido: 05-02-19

Revisado: 15-07-19

Modificado: 17-08-19

Aceptado: 01-09-19

\section{Bibliografía}

1. Fondo Colombiano de Enfermedades de alto Costo. Situación de la Enfermedad Renal Crónica, la Hipertensión Arterial y la Diabetes Mellitus en Colombia 2017. Bogotá: Fondo Colombiano de Enfermedades de alto Costo, 2018.

2. Angulo-Duplán $A$, Martínez-Martínez ML, Velázquez-Tlapanco J, Gallardo-Vidal LS, Camacho-Calderón N. Correlación entre la dependencia física

del paciente con Diálisis Peritoneal y la carga del cuidador de acuerdo a la funcionalidad familiar. Revista DIGITAL CIENCIA@UAQRO [Internet]. 2016 [Consultado 10 marzo 2016] (aprox. 9 p.). Disponible en: http://www.uaq.mx/investigacion/revista_ciencia@uaq/ArchivosPDF/v9-n1/MED-9.pdf.

3. Ambriz-López MC, Villalobos-Arámbula LM, Gallegos-Torres RM, Xeque-Morales AS, Alejandra M. Perfil del cuidador principal y su relación con el cuidado del personal de enfermería. Rev Enferm Inst Mex Seguro Soc 2014;22(3):123-9.

4. Jarillo Soto EC, Ramos del Río B. El cuidado informal de personas con enfermedad renal crónica. Una mirada desde la salud colectiva y la teoría de las representaciones sociales. En-clav. Pen 2014; 8(15):195-216.

5. Ruiz Antúnez ER, Poyo Poyo A, García Prieto $M L$, Pérez Yuste MP, Plaza García A, Martín Domínguez $\mathrm{V}$ y cols. Revisión de una Intervención de Enfermería. NIC: 007040 Apoyo al Cuidador Principal. Rev. enferm. CyL 2016;8(2):40-61.

6. Romero Massa E, Maccausland SeguraY, Solórzano Torrecila L. El cuidador familiar del paciente renal y su calidad de vida, Cartagena (Colombia). Salud Uninorte. Barranquilla (Col.) 2014;30(2):146-57.

7. Arechabala MC, Catoni MI, Palma E, Barrios S. Depresión y autopercepción de la carga del cuidado en pacientes en hemodiálisis y sus cuidadores. Rev Panam Salud Publica 2011;30(1):74-9.

8. Velázquez $Y$, Espín M. Repercusión psicosocial y carga en el cuidador informal de personas con insuficiencia renal crónica terminal. Rev Cub Salud Publica. 2014;40(1):3-17.

9. Noble H, Kelly D, Hudson P. Experiences of carers supporting dying renal patients managed without dialysis. J Adv Nurs. 2013;69(8):1829-39.

10. Bayoumi M. Subjective burden on family carers of hemodialysis patients. Open J Nephrol 2014;4(2): 79-85.

11. Eterovic C, Mendoza S, Sáez K. Habilidad de cuidado y nivel de sobrecarga en cuidadoras/es informales de personas dependientes. Enferm glob 2015;14(38):235-48. 
12. Gil I, Pilares JA, Romero R, Santos M. Cansancio del rol de cuidador principal del paciente en hemodiálisis. Enferm Nefrol. 2013;16(Supl1):S172-3.

13. Morton RL, Snelling P, Webster AC, Rose J, Masterson R, Johnson DW, Howard K. Dialysis modality preference of patients with CKD and family caregivers: a discrete-choice study. Am J Kidney Dis. 2012;60(1):102-11.

14. Contreras A, López Soto P, Crespo R. Análisis de la relación entre nivel de dependencia del paciente en hemodiálisis y sobrecarga del cuidador principal. Enferm Nefrol. 2014;17(2):98-103.

15. Méndez DA, González CRM, Mendoza GK. Identificación de enfermedad renal en sujetos aparentemente sanos, familiares de pacientes que acuden a hemodiálisis Rev Esp Med Quir 2012;17(1):29-33.

16. Celik G, Annagur BB, Yılmaz M, Demir T, Kara F. Are sleep and life quality of family caregivers affected as much as those of hemodialysis patients? Gen Hosp Psychiatry. 2012;34(5):518-24.

17. Williams LA. Burden, depressive symptoms, and perceived health in male caregivers of persons with end stage renal disease [Tesis]. Knoxville: The University of Tennessee;2015.

18. Da Silva Barreto $M$, Augusto da Silva MA, Sezeremeta DC, Basílio G, Silva Marcon S. Conhecimentos em saúde e dificuldades vivenciadas no cuidar: perspectiva dos familiares de pacientes em tratamento dialítico. Cienc Cuid Saude 2011; 10(4):722-30.

19. Rivero-García R, Segura-García E, Juárez-Corpus NB. Sobrecarga del cuidador primario de pacientes en programa de hemodiálisis generada por el reingreso hospitalario. Dial Traspl. 2015;36(2):72-7.

20. López D, Rodríguez L, Carreño S, Cuenca I, Chaparro L. Cuidadores de pacientes en diálisis peritoneal: experiencia de participar en un programa de habilidad de cuidado. Enferm Nefrol. 2015;18(3):189-95.

21. Shdaifat EA, Manaf MRA. Quality of life of caregivers and patients undergoing haemodialysis at Ministry of Health, Jordan. Int J Appl. 2012; 2(3): 78-85.
22. Aguilera AI, Castrillo EM, Linares $B$, Carnero $\mathrm{RM}$, Alonso AC, López $\mathrm{P}$ et al. Análisis del perfil y la sobrecarga del cuidador de pacientes en Diálisis Peritoneal y Hemodiálisis. Enferm Nefrol. 2016;19(4):359-65.

23. Aguilera A, Prieto M, González L, Abad B, Martínez $E$, Robles I et al. Una estrategia poco utilizada en el cuidado de pacientes con enfermedad renal crónica: la educación en grupo y multidisciplinar de pacientes y sus familiares. Enferm Nefrol. 2012;15(1):14-21.

24. Avşar U, Avşar UZ, Cansever Z, Yucel A, Cankaya $E$, Certez $H$ et al. Caregiver Burden, Anxiety, Depression, and Sleep Quality Differences in Caregivers of Hemodialysis Patients Compared With Renal Transplant Patients. Transplant Proc. 2015;47(5):1388-91.

25. Carmona C, Nolasco C, Navas L, Caballero J, Morales $P$. Análisis de la sobrecarga del cuidador del paciente en diálisis peritoneal. Enferm Nefrol 2015;18(3):180-8.

26. Guacas Guerra MA. Carga del cuidado de los cuidadores de personas con enfermedad renal crónica, sometidos a diálisis y hemodiálisis en una Unidad renal del municipio de Pasto en el periodo marzo-julio de 2014. leu [Internet]. 1 [Consultado 13 ago 2017]; Disponible en: http://ojseditorialumariana.com/index.php/libroseditorialunimar/article/ view/1021.

27. Rodríguez Chancafe AM. Conocimiento sobre medidas preventivas y factores sociodemográficos en el familiar cuidador del paciente con enfermedad renal crónica. [Tesis] Universidad nacional de Trujillo. 2017. [Consultado 14 ago 2017]. Disponible en: http://dspace.unitru.edu.pe/handle/UNITRU/14458.

28. Alnazly E. Coping strategies and socio-demographic characteristics among Jordanian caregivers of patients receiving hemodialysis. Saudi Journal of Kidney Diseases and Transplantation. 2016; Jan 1;27(1):101.

29. Arechabala MC, Catoni MI, Barrios S, Palma E. Validación al español de la escala de auto percepción de carga de cuidado. Acta Paulista de Enfermagem. 2012;25(1):140-5. 
30. Mejía LD, López L. La familia y la cultura: una conexión innovadora para el cuidado de la salud. Index Enferm. 2010; 19(2-3):138-42.

31. Flores GE, Rivas RE, Seguel PF. Nivel de sobrecarga en el desempeño del rol del cuidador familiar de adulto mayor con dependencia severa. Cienc enferm 2012;18(1):29-41.

32. Carrillo G, Chaparro L, Sánchez B. conocimiento y acceso a las TIC en personas con enfermedad crónica y cuidadores familiares en Colombia. Enferm. Glob. 2014;13(2):123-34.

33. Carrillo GM, Chaparro L, Sánchez B. Carga del cuidado en cuidadores familiares de personas con enfermedad crónica en la región amazónica de Colombia. Med UPB 2014;33(1):26-37.

34. Pinto XV, Moreno SC, Díaz L. Factores que influencian la habilidad y sobrecarga del cuidador familiar del enfermo crónico. Rev Univ Ind Santander Salud. 2017;49(2):330-8.

35. Guerra-Martín MD, Amador-Marín B, Martínez-Montilla JM. Problemas de salud de los cuidadores familiares de personas mayores de 65 años afectadas de insuficiencia renal crónica: una revisión sistemática. Anales Sis San Navarra. 2015; 22(3):425-38.
36. Walker RC, Hanson CS, Palmer SC, Howard K, Morton RL, Marshall MR, Tong A. Patient and caregiver perspectives on home hemodialysis: a systematic review. American Journal of Kidney Diseases. 2015 Mar 1;65(3):451-63.

37. Galvis C, Aponte L, Pinzón M. Percepción de la calidad de vida de cuidadores de pacientes asistentes a un programa de crónicos, Villavicencio, Colombia. Aquichan. 2015;16(1):23-34.

38. Ballesteros JE, Rodríguez AM, Cantor M, Peñalosa G, Valcárcel E. Caracterización y percepción de carga de cuidadores familiares de personas con enfermedad crónica en Arauca. Rev Orinoquia. 2015;19(1):100-5.

39. Ávila JH, Vergara M. Calidad de vida en cuidadores informales de personas con enfermedades crónicas. Aquichan 2014;14(3):417-29.

40. Murillo Moreno LM. Necesidades y motivaciones de cuidadores familiares de pacientes con enfermedad renal crónica (Doctoral dissertation, Corporación Universitaria Minuto de Dios).2018.

Este artículo se distribuye bajo una Licencia Creative Commons Atribución-NoComercial 4.0 Internacional. https://creativecommons.org/licenses/by-nc/4.0/

\section{Open Access (c) () ()}

